http://jmscr.igmpublication.org/home/ ISSN (e)-2347-176x ISSN (p) 2455-0450

crossref DOI: https://dx.doi.org/10.18535/jmscr/v7i10.46

Journal Of Medical Science And Clinical Research

Original Article

\title{
Jaundice as sign of Urinary Tract Infection in Early Infancy: A hospital based Observational study
}

\author{
Authors \\ Neupane Shankar ${ }^{1 *}$, Sharma Arun Kumar ${ }^{2}$, Shrestha Laxman ${ }^{3}$, Subedi Sonika \\ ${ }^{1}$ Department of Pediatrics, Shankarapur Hospital, Jorpati, Kathmandu, Nepal \\ ${ }^{2,3}$ Department of Child Health, Tribhuvan University Teaching Hospital, Maharajgunj, Kathmandu, Nepal \\ ${ }^{4}$ Emergency Department, Shankarapur Hospital, Jorpati, Kathmandu, Nepal \\ *Corresponding Author \\ Neupane Shankar \\ Shankarapur Hospital, Kathmandu, Nepal
}

\begin{abstract}
Background: Neonatal jaundice is a common problem and often warrants extensive evaluation. Bacterial infections in neonates including urinary tract infection (UTI) can manifest with jaundice. Guidelines on management of afebrile, asymptomatic jaundiced infants do not necessarily include evaluation of UTI. The aim of this study was to determine the incidence of UTI in asymptomatic, jaundiced infants younger than 8 weeks old.

Methods: Significantly jaundiced infants aged 4 days to 8 weeks and born at $\geq 35$ weeks of gestation who were otherwise well in outpatient and inpatient settings were prospectively included in the study. Infants were significantly jaundiced if they had jaundice visualized up to Kramer's dermal zone 4 or 5 in first two weeks of life and/or jaundice prolonged beyond first two weeks of age. All significantly jaundiced infants underwent suprapubic aspiration for urine collection and UTI was diagnosed with isolation of any organism in the sample. A serum fractionated bilirubin level was obtained on all cases. All infants underwent other relevant investigations as indicated. A standardized questionnaire was completed to determine the relationship with demographic features and prenatal, intranatal, and postnatal events.

Results: One hundred and twenty-six infants, 81 (64.3\%) of whom were boys were enrolled at a mean age of 16 \pm 14 days. Most of the neonates $(118,93.7 \%)$ had onset of jaundice within 4-14 days of life. Only one out of 126 infants $(0.8 \%)$ had a diagnosis of UTI, Escherichia coli was isolated as a causative organism. Etiology of jaundice was established as breast milk jaundice in majority of infants (122, 96.8\%). Since there was a single patient with UTI, the influence of various risk factors upon incidence of UTI could not be analyzed.

Conclusion: A very low incidence of UTI was identified in infants with jaundice. The study failed to show significant association between jaundice and UTI. Routine evaluation for UTI may not be suggested in asymptomatic jaundiced neonates. Further studies in larger population should be performed to determine if newborns with asymptomatic jaundice require routine evaluation for UTI.

Keywords: Jaundice, Urinary Tract Infection, Infant, Kramer's dermal zone.
\end{abstract}




\section{Introduction}

Jaundice is common problem during the newborn period, between $60 \%-80 \%$ of healthy infants can present with idiopathic neonatal jaundice. ${ }^{1}$ Unexplained jaundice may be associated with bacterial infections in newborn, such as Urinary Tract Infection (UTI). ${ }^{2}$

UTI is reported in up to $7.5 \%$ of asymptomatic, afebrile, jaundiced infants younger than 8 weeks of age. ${ }^{2}$ The incidence of UTI varies from 0.1 to $1 \%$ among neonates. ${ }^{3,4}$ UTI is considered to be a relatively common cause of prolonged jaundice, urine culture should be considered in the evaluation of jaundiced infants older than three days of age with an unknown etiology. ${ }^{5}$ The incidence of urinary tract abnormalities in infants with UTI is between $30 \%$ and $55 \%$, the most common being vesicoureteric reflux. ${ }^{6-11}$ Renal scarring, hypertension, and even kidney failure can be prevented by early diagnosis and treatment of UTI. ${ }^{12}$ Current guidelines do not recommend evaluation for UTI among babies with hyperbilirubinemia. ${ }^{13}$

\section{Materials and Methods}

This hospital based prospective observational study was conducted in pediatric Out Patient Department (OPD), pediatric ward, maternity ward, immunization clinic and Neonatal Intensive Care Unit (NICU) of Tribhuvan University Teaching Hospital(TUTH), Kathmandu for a period of 12 months from October 2013 to October 2014. The Institutional Review Board of TUTH approved the study design and written informed consent was obtained from caregivers of all cases.

\section{Study population}

Newborns aged between 4 days to 8 weeks of life who were born at term and near term beyond 35 weeks of gestation with clinically significant jaundice in the absence of other symptoms of illness were eligible for study. Clinically significant jaundice was defined as extension of clinical jaundice to Kramer's dermal zone 4 or 5 in first two weeks of life or with prolonged jaundice beyond first two weeks of life. Newborns were excluded if preterm $<35$ weeks of gestation at birth, jaundice appeared within 72 hours of life, inability to obtain consent from caregivers and if jaundice presented with other symptoms such as fever, vomiting, diarrhea, poor feeding, tachypnea, lethargy, or irritability. The newborns were screened for the presence of jaundice. Newborns were examined to determine the clinical extent of jaundice using Kramer's dermal zone. Newborns were completely evaluated to determine if they had any other abnormality and pertinent findings of clinical relevance.

\section{Historical Data}

Information regarding prenatal and intranatal events, such as gestational age, maternal blood group, maternal infections, prolonged rupture of membranes, pregnancy complications, mode of delivery, and newborn sex were collected as per the study pro forma. Postnatally newborns were examined for features of neonatal sepsis. Information regarding onset and progression of jaundice and feeding practice were noted.

\section{Clinical Procedures}

Suprapubic aspiration was performed in all babies with significant jaundice to obtain urinary sample. Urine was sent for urinalysis, microscopy, Gram stain and culture. Centrifuged urine sample was examined microscopically under high power field (HPF) for White Blood Cells (WBC) more than five reported as pyuria, Red Blood Cells (RBC), bacteriuria, albumin and epithelial cells. Specimen of urine were sent for quantitative urine culture and considered positive if a single pathogen was isolated. It was then subjected to biochemical tests to confirm the pathogens. Counting of colonies was done by standard microbiologic techniques. After isolation of organism, the isolate was subjected to anti biogram study on Muller Hilton agar by Kirby - Bayers disc diffusion method. By measuring the zone of inhibition with the reference of standard table, the results were classified into sensitive / intermediate sensitive and resistant for each antibiotics tested. A serum fractionated bilirubin level was obtained on all 
cases. All relevant investigations were carried out as guided by history and clinical examination findings as per institution protocol.

\section{Disposition and Follow-up}

After an initial evaluation, patients were either admitted to the hospital for phototherapy and/or underwent a sepsis evaluation if sepsis was suspected or discharged from the hospital to follow-up with the reports. A full septic work up was carried in those with clinical features of sepsis and treatment started immediately. Standard of necessary care were continued depending on case. Patients with positive urine culture were contacted and instructed to return to the hospital for further evaluation and management of UTI. The sepsis evaluation included a complete blood cell count with peripheral smear, C-reactive protein and blood culture. Cases with direct hyperbilirubinemia underwent relevant investigations and received treatment as per hospital protocol. Cases with indirect hyperbilirubirubinemia in the phototherapy range or exchange transfusion range as defined by American Academy of Pediatrics guidelines received appropriate therapy. Subsequent visits after 3 and 7 days to evaluate for jaundice progression was advised for these babies. Patients admitted in ward and neonatal unit were followed during the hospital stay.

Statistical Analysis: Sample size estimates were based on an average study population patient flow to hospital and corrected for finite population. Data was collected, tabulated and analyzed using SPSS software version 20. Descriptive data were reported as mean \pm standard deviation and associations between categoric variables were analyzed using the Fischer's exact test and nonparametric tests were analyzed with MannWhitney $U$ test. Statistical significance was defined as a $\mathrm{P}$ value $<0.05$.

\section{Results}

\section{Study Population and Demographics}

A total of 1195 cases in the age group of 4 days to 8 weeks were screened for the presence of jaundice. Of these, 401 cases had jaundice at presentation and 126 cases met the inclusion criteria and were enrolled in the study. Of 126 cases, 52 babies were enrolled from OPD, 50 from wards, 16 from immunization clinic and 8 from NICU. Among enrolled cases 70 babies were evaluated for clinically significant jaundice based on Kramer's dermal zone, 48 babies were evaluated for prolonged jaundice and 8 babies were evaluated for possibility of sepsis. The mean age of the study population was $16.17 \pm 14.76$ days with a range of 4-56 days and interquartile range of 5-23.5 days. The mean birth weight was $2.829 \pm 0.502 \mathrm{~kg}$ and ranged from $1.5 \mathrm{~kg}$ to $4.3 \mathrm{~kg}$. One hundred five cases $(83.3 \%)$ were born at 37 42 weeks, 20 cases $(15.9 \%)$ between 35-37 weeks and 1 case $(0.8 \%)$ beyond 42 weeks. Seventy one cases $(56.4 \%)$ were born by spontaneous vaginal delivery, 52 cases $(41.2 \%)$ by cesarean section and 3 cases $(2.4 \%)$ by assisted vaginal delivery. Eighty one infants $(64.3 \%)$ were males and 45 infants $(35.7 \%)$ were females. None of the boys were circumcised. Six cases $(4.8 \%)$ were small for gestational age. Ninty seven percent of babies were exclusively or predominantly breastfed.

Majority of infants had onset of jaundice between 4-14 days of life as shown in table 1.

Table 1 Time of onset of jaundice

\begin{tabular}{|l|c|c|}
\hline Jaundice onset & Frequency & Percentage \\
\hline 4-14 days & 118 & 93.7 \\
\hline$>14$ days & 8 & 6.3 \\
\hline Total & 126 & 100 \\
\hline
\end{tabular}

The mean total bilirubin level of the study population was $16.09 \pm 6.08 \mathrm{mg} / \mathrm{dl}$. Mean direct bilirubin level was $1.52 \pm 1.34 \mathrm{mg} / \mathrm{dl}$. Seven babies $(5.6 \%)$ had direct bilirubin value $>20 \%$ of total serum bilirubin value.

The findings of urinalysis are as shown in table 2.

Table 2 Urinalysis results

\begin{tabular}{|l|c|c|c|}
\hline Urine RME & Median & Range & IQR \\
\hline WBC per HPF & 2.00 & $0-6$ & $2-3$ \\
\hline RBC per HPF & 0.00 & $0-25$ & $0.00-0.00$ \\
\hline
\end{tabular}

95 cases $(75.4 \%)$ had no albuminuria and 22 cases $(17.4 \%)$ had trace albumin in urine. 


\section{Incidence of UTI}

Positive urine culture was found in a single case with a rate of $0.8 \%$ as shown in figure 1.

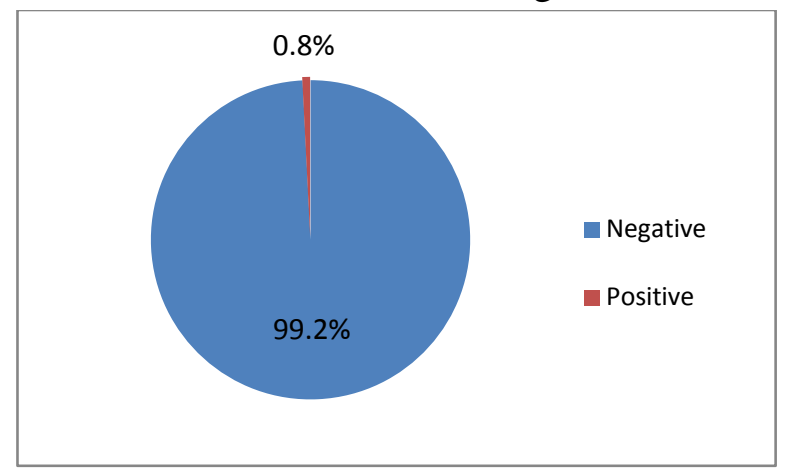

Figure 1: Incidence of Urine Culture
The baby with a positive urine culture was 45 day old male infant. The mean age of babies with negative urine culture was $15.49 \pm 14.59$ days. There was no statistically significant difference in incidence of UTI among male and female babies. Similarly there was no statistical difference in incidence of UTI with respect to age as shown in table 3 .

Table 3 Comparison of age and sex with positive and negative urine cultures

\begin{tabular}{|c|c|c|c|}
\hline \multirow{2}{*}{ Characteristics } & \multicolumn{2}{|c|}{ Urine Culture } & p-value \\
\cline { 2 - 4 } & Positive $(\mathrm{n}=1)$ & $\begin{array}{c}\text { Negative } \\
(\mathrm{n}=125)\end{array}$ & \\
\hline Age (Days) & \multicolumn{2}{|c}{} & Mann-Whitney U test \\
\hline Mean (SD) & $45.00(0.00)$ & $15.49(14.598)$ & 0.190 \\
\hline Gender & \multicolumn{2}{|c|}{1.000} \\
\hline Male & 1 & 80 & \multirow{2}{*}{ Fisher's Exact test p value } \\
\hline Female & 0 & 45 & \\
\hline
\end{tabular}

No statistically significant difference in incidence of UTI existed among babies with regard to birth weight, gestational age, mode of delivery, age of onset of jaundice, total \& direct bilirubin values as shown in table 4.

Table 4 Relationship between type of hyperbilirubinemia and UTI

\begin{tabular}{|c|c|c|c|c|c|}
\hline \begin{tabular}{|l} 
Direct and Total \\
Bilirubin
\end{tabular} & $\begin{array}{c}\text { Urine culture and } \\
\text { sensitivity }\end{array}$ & $\mathrm{N}$ & Mean & Std. Deviation & $\begin{array}{c}\text { Mann Whitney U Test } \\
\text { p-value }\end{array}$ \\
\hline \multirow[t]{2}{*}{ Total bilirubin mg/dl } & Positive & 1 & 9.8800 & 0.000 & \multirow[b]{2}{*}{0.333} \\
\hline & Negative & 125 & 16.1463 & 6.08607 & \\
\hline \multirow[t]{2}{*}{ Direct bilirubin mg/dl } & Positive & 1 & 0.5100 & 0.000 & \multirow{2}{*}{0.111} \\
\hline & Negative & 125 & 1.5354 & 1.34970 & \\
\hline
\end{tabular}

\section{Outcomes}

Patients were followed for the progression or resolution of jaundice and/or development of new symptoms. Only one baby had UTI. Thyroid hormone supplementation was begun on two babies with hypothyroidism. There was resolution of jaundice and/or reduction in intensity of jaundice in sequential follow up visits in rest 123 cases. Majority of these latter cases were either exclusively or predominantly breastfed attributing their jaundice to breast milk jaundice.

One patient with positive urine culture had isolation of Escherichia coli as a single pathogen.
The organism was sensitive to nitrofurantoin, cefixime and norfloxacin however was resistant to ampicillin and amoxicillin. This 45 day uncircumcised male infant presented for routine immunization, jaundice was an incidental finding. Antenatal ultrasonography was normal. This baby was born to primigravida mother at 40 weeks of gestation via spontaneous vaginal delivery weighing $3.5 \mathrm{~kg}$. This baby was exclusively breastfed. Jaundice was noted since $5^{\text {th }}$ day of life. Physical examination was unremarkable except for jaundice. This baby was evaluated for prolonged jaundice at presentation. Investigation 
revealed total serum bilirubin value of $9.88 \mathrm{mg} / \mathrm{dl}$ with direct bilirubin of $0.51 \mathrm{mg} / \mathrm{dl}$. Urinalysis revealed pus cells of 2-4 WBC/HPF, urine RBCnil and absent albumin. Mother was blood group B positive. Complete blood count was normal, peripheral blood smear- normocytic normochromic. Glucose-6-Phosphate Dehydrogenase level was normal. Thyroid function test was normal. Following positive urine culture, this case was contacted to follow up for further management and evaluation. However this case declined to follow up.

\section{Discussion}

Bacterial infection in newborns can present as jaundice, and association of jaundice with UTI has been reported by several studies. ${ }^{5,14}$ The clinical manifestations of UTI in newborn infants are extremely variable, ranging from severe illness to nonspecific signs and symptoms, such as growth failure, poor feeding, vomiting, diarrhea, fever, irritability, lethargy, abnormal urination with oliguria, polyuria or malodorous urine, and jaundice. $^{15}$

In the present study, UTI was found in $0.8 \%$ of asymptomatic jaundiced infants younger than 8 weeks old. This is in sharp contrast to other previous studies. UTI is reported in $7.5 \%$ of asymptomatic, afebrile, jaundiced infants younger than 8 weeks old. $^{2}$ Other studies showed prevalence between $5 \%$ and $11 \%$ among febrile infants younger than 8 weeks of age. ${ }^{16-19}$

The difference may account in part by the population group studied. Majority of previous studies are based on study of white babies., ${ }^{2,14}$ UTI rates are higher among white infants $8 \%$ than among black infants $4.7 \%$ reflecting racial/ethnic variation in prevalence of UTI. ${ }^{18,20}$ The age group of babies was between 4 days to 8 weeks of age in our study with mean age of 16 days. However in other studies this age group was not uniform some were studied in first two weeks of age ${ }^{5,21}$ while in others it was beyond two weeks of age. ${ }^{22}$ In previous studies cases were enrolled from newborn nursery ${ }^{5,22}$ and from emergency department $^{2}$ where clinical profile of patients might be different whereas in our study babies were mostly enrolled from OPD, wards and immunization clinic which could have accounted for the difference in UTI.

Breast feeding has been considered protective for UTI. $^{23}$ Majority of babies were formula or mixed fed in previous studies. ${ }^{2,14}$ Our study comprised predominantly breast fed babies with indirect hyperbilirubinemia. High rate of breast feeding in our setting means that prevalence of UTI is likely to be different. Significant elevation in unconjugated bilirubin (breast milk jaundice) develops in an estimated $2 \%$ of breast-fed term infants after the 7 th day of life. ${ }^{20}$ Jaundiced breastfed infants who are well are unlikely to have serious disease. $^{24}$

This difference might also be attributed to method of urine collection wherein urine was collected by bladder catheterization in previous studies ${ }^{25}$ whereas in our study all samples were collected by suprapubic aspiration. Contamination rates vary with method of urine collection, $26 \%$ in clean catch urine, $12 \%$ in catheter sample urine and $1 \%$ in suprapubic aspiration. ${ }^{25}$

In our study UTI was found in a 45 day old uncircumcised male infant. Boys predominate among infants with UTI during the first 3 months of life. ${ }^{6,8}$ Among febrile male infants, less than 3 months of age, $2.4 \%$ of circumcised males and $20.1 \%$ of uncircumcised males have a UTI. ${ }^{26,27}$ The pathogen isolated was E. coli in our study which is a common organism reported in previous studies. ${ }^{25}$ E. coli is responsible for the vast majority of UTI in infants younger than three months of age. ${ }^{28}$

In breastfed infants, jaundice can persist beyond 10 to 14 days of age. ${ }^{29}$ The incidence of UTI among babies with prolonged jaundice was $6 \% .^{(22)}$ In our study, 48 babies (38\%) had prolonged jaundice and one of these babies had UTI with incidence of $2.08 \%$. Prolonged jaundice is more common in breastfed infants than artificially-fed infants. ${ }^{30}$ High rate of breast feeding practice in our setting means that 
prevalence of prolonged neonatal jaundice is likely to be different. Although UTI has been identified as an important risk factor for prolonged neonatal jaundice, ${ }^{22}$ high prevalence of breast milk jaundice in breastfed infants in our setting might actually mean that UTI may not be as important as in other studies.

UTI can occur without apparent signs, and jaundice may be an important and sometime the presenting feature of UTI. ${ }^{31}$ The case with UTI in present study had total bilirubin value of 9.88 $\mathrm{mg} / \mathrm{dl}$ with bilirubin fraction of $0.51 \mathrm{mg} / \mathrm{dl}$ $(5.16 \%)$ revealing indirect hyperbilirubinemia. Newborn infants with UTI present with unconjugated hyperbilirubinemia in the early stages but conjugated hyperbilirubinemia after 6 weeks. $^{32}$ The hyperbilirubinemia associated with UTI can be unconjugated and related to hemolysis caused by E coli. ${ }^{33,34}$ Garcia et al reported that patients with an elevated conjugated bilirubin fraction were likely to have UTI, ${ }^{2}$ the case with positive UTI did not have high direct bilirubin level.

The incidence of neonatal UTI varies from $0.1 \%$ to $1 \%$ in the general population of healthy newborns. ${ }^{35}$ Previous studies by Bourchier et al, ${ }^{7}$ Edelman et al, ${ }^{36}$ Drew et al, ${ }^{37}$ and Wettergren et $\mathrm{al}^{38}$ have found the incidence of bacteriuria in asymptomatic neonates to be between 0.5 and $1 \%$. This is in contrast to studies where incidence of UTI was several times higher (5 to 11 times) in jaundiced babies at ages up to 8 weeks of age. $^{2,5,14,22,39}$ Our finding of UTI incidence of $0.8 \%$ was closely reflecting incidence of UTI among healthy newborns.

In our study with positive UTI, urinalysis revealed absence of pyuria. Crain and Gershel, ${ }^{17}$ in a prospective study of 430 febrile infants younger than 8 weeks old, identified 33 with a UTI. Only $48 \%$ of infants had an abnormal urinalysis, defined as 5 or more WBC/HPF or visible bacteria on microscopic examination of unspun urine. ${ }^{17}$ Similar studies by Landau et al reported that 31 (28\%) of 128 infants younger than 16 weeks with a UTI, had fewer than $5 \mathrm{WBC} / \mathrm{HPF}$ in the urine. ${ }^{40}$
Antenatal USG revealed no urinary tract anomaly in our case with positive UTI. In a study of infants younger than 8 weeks old diagnosed with a UTI, $22(49 \%)$ of 45 infants had urinary tract abnormalities that included hydronephrosis, renal atrophy, and vesicoureteral reflux. ${ }^{41}$

Our study had several limitations. All eligible cases could not be enrolled for study. UTI was diagnosed in one case, therefore statistical calculations to determine the risk factors could not be done. The case with positive UTI could not be evaluated for urinary tract anomalies with imaging studies as the case was lost to follow up. All the cases did not undergo uniform set of investigations apart from urine and fractionated bilirubin. Those who presented before 14 days of life were not offered thyroid testing which was reserved as workup for prolonged jaundice. Septic work-up was done for those with clinical features suggestive of sepsis. Larger sample size studies are recommended to determine the true differences in UTI prevalence.

\section{Conclusion}

UTI can occur in asymptomatic jaundiced infant younger than 8 weeks of age. In population with a high rate of breastfeeding breast milk jaundice is common, other diagnosis like UTI is uncommon. Routine evaluation for UTI may not be suggested in asymptomatic jaundiced neonates. Further large scale population studies should be performed to determine if newborns with asymptomatic jaundice require routine evaluation for UTI.

Conflict of interest: No conflict of interest to declare.

\section{Source of funding: None}

\section{Acknowledgements}

We thank the newborns and caregivers of newborns who participated in this study. We express our gratitude to all the staffs of the hospital who contributed to the study. 


\section{References}

1. Chou RH, Palmer RH, Ezhuthachan S et al. (2003). Management of hyperbilirubinemia in newborns: measuring performance by using a benchmarking model. Pediatrics, 112: 1264-73.

2. Garcia FJ, Nager AL. Jaundice as an early diagnostic sign of urinary tract infection in infancy. Pediatrics. 2002 May; 109(5):846-51.

3. Elder JS. Urologic disorder in infants and children In Behrman, Kleigman, eds. Nelson Text Book of Pediatrics. Philadelphia; WB. Saunders, 2004; 18121814.

4. Maisels MJ. Mixed forms of jaundice In Mac Donald, ed. Avery's Neonatology Pathophysiology \& Management of the Newborn. Lippincott; Williams \& Wilkins, 2005; 807-808.

5. Bilgen H OE, Unver T, Biyikli N, Alpay $\mathrm{H}$, Cebeci D. Urinary tract infection and hyperbilirubinemia. Turk J Pediatr. 2006;48:51-5.

6. Ginsburg CM MG. Urinary tract infections in young infants. Pediatrics. 1982;69:40912.

7. Bourchier D AG, Maling TMJ. Radiological abnormalities in infants with urinary tract infections. Arch Dis Child. 1984;59:620-4.

8. Ring E ZG. Urinary infection and malformations of urinary tract in infancy. Arch Dis Child. 1988;63:818-20.

9. Tsingoglou $\mathrm{S}$ DJ. Lower urinary obstruction in infancy. Arch Dis Child. 1972;47:215-7.

10. Rushton HG MM. Dimercaptosuccinic acid renal scintigraphy for the evaluation of pylonephritis and scarring: a review of experimental and clinical studies. J Urol. 1992;148:1726-32.

11. Greenfeld SP WJ. Vesicoureteral reflux: practical aspects of evaluation and management.

Pediatr

Nephrol.

1996;10:189-794.

12. Struthers S SJ, Parker K, Goddard J, Hallett R. Parental reporting of smelly urine and urinary tract infection. Arch Dis Child. 2003;88:250-2.

13. Robertson WO, lmquist JR, Light IJ, Maisels MJ et al. American Academy of Pediatrics, Provisional Committee for Quality Improvement, Practice parameter: management of hyperbilirubinemia in the healthy term newborn. Pediatrics 199; 94(4): 558-65.

14. Shahian M, Rashtian P, Kalani M .Unexplained neonatal jaundice as an early diagnostic sign of urinary tract infection. Int J Infect Dis 2012; 16 (7):487-90.

15. JM L. Sixty-six infants with urinary tract infection in first month of life. Arch Dis Child. 1972;47:218-26.

16. Krober MS BJ, Powell JM, Smith FR, Seto DS. Bacterial and viralpathogens causing fever in infants less than 3 months old. Am J DisChild. 1985;139:889-92.

17. Crain EF GJ. Urinary tract infections in febrile infants younger than 8 weeks of age. Pediatrics. 1990;86:363-7.

18. Hoberman A CH, Keller DM, Hickey R, Davis H, Ellis D. Prevalence of urinary tract infection in febrile infants. J Pediatr. 1993;123:17-23.

19. Hoberman A WE. Urinary tract infections in young febrile children. Pediatr Infect Dis J. 1997;16:11-7.

20. Lawrence M. Gartner IMA. Studies of prolonged neonatal jaundice in the breastfed infant. The Journal of Pediatrics 1966;68(1):54-6.

21. Mutlu M, Cayır Y, Aslan Y. Urinary tract infections in neonates with jaundice in their first two weeks of life. World $\mathbf{J}$ Pediatr. 2014 May; 10(2):164-7.

22. Nader Pashapour AAN, Sariyeh Golmohammadlou. Urinary Tract Infection in Term Neonates with 
Prolonged Jaundice. Pediatric Urology Journal 2007;4(2).

23. Kring MJMaE. Risk of Sepsis in Newborns With Severe Hyperbilirubinemia. Pediatrics. 1992;90:741.

24. Hannam S, McDonnell M, Rennie JM. Investigation of prolonged neonatal jaundice. Acta Paediatr 2000;89(6):694-7.

25. Tosif S BA, Oakley E, Donath S, Babl FE. Contamination rates of different urine collection methods for the diagnosis of urinary tract infections in young children: an observational cohort study. J Paediatr Child Health. 2012;48(8):659.

26. McCandless DW. History of Bilirubin Kernicterus. Pediatrics. 2011 (XIV):286.

27. Joseph J. Zorc DALea. Clinical and Demographic Factors Associated With Urinary Tract Infection in Young Febrile Infants. Pediatrics. 2005;116:644.

28. Schalger TA (2001). Urinary Tract Infections in Children Younger Than 5 Years of Age. Paediatric Drugs 3(3): 219227.

29. Kivlahan C JE. The natural history of neonatal jaundice. Pediatrics. 1984;74:364-70.

30. Crofts DJ, Michel VJ-M et al. Assessment of stool colour in community management of prolonged jaundice in infancy. Acta Pediatr 1999;88:969-74.

31. Long SS KJ. Infectious Diseases of the Fetus and Newborn Infant 5th edition ed: WB Saunders; 2001.

32. Lee HC FS, Yeung CY, Tsai JD. Urinary tract infections in infants: comparison between those with conjugated vs unconjugated hyperbilirubinaemia. Ann Trop Paediatr. 2005;25:277-82.
33. Thurman WG. "Changes in red cell fragility with infection." American Journal of Diseases of Children. 1960;100(4).

34. SEELER RA. Urosepsis With Jaundice Due to Hemolytic Escherichia coli Am J Dis Child. 1973;126(3):414.

35. Briton L SL, Edelman CM. Neonatalprenatal medicine. London: Mosby; 2002.

36. Edelman CM OJ, Fine BP, Martinez AB. The prevalence of bacteriuria in full-term and premature newborn infants. J Pediatr. 1973;82:125-32.

37. Drew JH AC. Radiological findings in newborn infants with urinary infection. Arch Dis Child. 1976;51:628-30.

38. Wettergren B JU, Jonasson G. Epidemiology of bacteriuria during the first year of life. Acta Paediatr Scand. 1985;74:925-33.

39. Ghaemi S FR, Kelishadi R. Late onset jaundice and urinary tract infection in neonates. Indian J Pediatr. 2007;74(2): 139-41.

40. Landau D TM, Brennan J, Majd M. The value of urinalysis in differentiating acute pyelonephritis from lower urinary tract infection in febrile infants. Pediatr Infect Dis J. 1994;13:777-81.

41. Goldman M LE, Strauss S, et al. Imaging after urinary tract infection in male neonates. Pediatrics. 2000;105:1232-5. 